\title{
A Research on Energy Efficient Multiple Query Optimization in WSN
}

\author{
Santosh Kumar Sahoo, Ajit Kumar Nayak
}

\begin{abstract}
Back in the last couple of decades, wireless sensor networks (WSNs) have researched different applications are as from personalized, marketplace, armed forces, agriculture, and environment tracking into Web Things (IoT). The detector node's battery ability gets an essential issue if it isn't feasible to recharge or replace battery life. Inside this newspaper, we've reported that an extensive evaluate on hierarchical routing protocols predicated on single/multi-hop communicating and also for homogenous/heterogeneous WSNs together with a contrast of unique present protocols has been completed about the grounds of several elements which are energy intake, finishing delay, and load balancing, system equilibrium and scalability. A few facets which help determine the creation and the plan of navigation protocols may also be talked about. Some benefits and pitfalls of every protocol have were all appreciated.
\end{abstract}

\section{INTRODUCTION}

The software of Wireless sensor networks (WSNs) have grown broadly while within the last number of decades. A wireless sensor system is actually really just a system of sizable numbers of sensor nodes located to do task. The role of every and every detector node would be always to track and also accumulate the info out of the nearby environment and then ship it into the bottom station (BS) after a particular protocol. The BS functions like a gateway between your tracked place along with the ending server or user. In the beginning, WSNs had been employed for surveillance in the conflict subject or alternative military software [1 3 ] these as intrusion detection system (IDS), however today they're used too in many different areas that range from soil sensing [4], to promote food and agriculture [5 6], very low priced transport [7 8 ] and home-automation [9], medical maintenance. Earth observation includes air pollution tracking, fire detection [10 11 ], landslide detection and drinking water quality tracking, flooding detection etc.. Industrial programs involve machine wellness tracking and wastewater tracking, datalogging and rapid crisis reply etc.. Even in healthcare tracking, particular wearable apparatus are all built with detector nodes plus they are sometimes implanted straight into the body for all-around observation called human anatomy space system. The installation of sensor nodes in to the area under analysis might be ordered or randomized making use of mathematical equipment like arbitrary charts. Particular other installation problems include coverage and connectivity metrics whether it's application special. About the flip side, WSNs conveys some significant inescapable advantages. Primarily they are

Revised Version Manuscript Received on 10 September, 2019.

Santosh Kumar Sahoo, Department of Computer Science \& Engineering, Trident Academy of Technology, Bhubaneswar, Odisha, India. (Email: researchsub01@gmail.com)

Ajit Kumar Nayak, Department of IT, Siksha O Anusandhan Deemed to be University, Odisha, India.

(Email: researchsub01@gmail.com) embraced within the locations that are nearly inaccessible and second, their system topology isn't regarded. This produces the detector node's source shrouded in character so that consequently, their vitality may not be properly used. As a result of such limitations and electricity conservation is getting an integral and leading condition as as a result the functioning life of the general system might be raised. For a number of the normal wireless network issues, scientists are employed to fulfill with up with the power conservation conditions. All these are known for: discovering affordable methods to WSN issues. A few procedures to fit with up with the power efficiency prerequisites are improved so much before now. More than a few of those include processes like moderate access management, routing, Self Organization and bandwidth sharing etc.. Concurrently, these processes needs to think about a stability energy efficacy, precision and latency with routine architectures therefore the life of the network has been protracted. Even though for conventional wireless networks, lots of protocols and algorithms are suggested. Sensor networks exude a few extra characteristics that create sure they are equivalent to platforms that are intermediate. Sensor networks include substantially high multitude of detector nodes when compared with adhoc networks, that are densely set up and also are somewhat more prone to failures [3 4 ]. In case the detector nodes are thought of as portable, subsequently for special applications to that the topology of some detector network's varies and too frequently. The design used with the detector nodes in WSNs is chiefly an broadcast communicating comprising pointtopoint communications established almost all of the social networks. Sensor nodes have about 3 limits : memory, electrical electricity and also their power of computation plus so they contain of great number of costs plus detector. Consequently, they might possibly perhaps well not need worldwide identification (IDs). The majority of the current protocols and algorithms aren't exactly ideal worthy of detector networks simply because they've got distinct application prerequisites and also execute certain specific capabilities.Sensor nodes expend most of the vitality throughout communicating. The others of this really is absorbed throughout processing and sensing operation. Even the annihilation with the inefficiency in the least levels of the protocol pile of communicating at WSN is demanded. Notably at the network coating, 1 ought to invent a number of innovative electricity effective routing methods which offer steady data communicating on the list of installed sensor nodes along with outside base channel to lengthen the system 
life. At community coating, lots of procedures have been completely accompanied with the investigators prior to developing power efficient routing strategies viz. Flat routing, hierarchical routing, location-based routing etc.. [1 3 ] and one of these procedures hierarchical navigation seems are absolutely the absolute most appealing as most calculations are improved fixing such like being a foundation.

\section{SENSOR NODE COMPONENTS}

The diagrammatic representation of a sensor node is done in Fig.1 [12] A detector node consists of 4 segments: detection device, processing transceiver device and electrical strength device. The branch is application-dependent. A few of the elements are location-finding method, strength generator and mobilizer. Sensing components include 2 standard parts or subunits: Patches and analog-to-digital converters (ADCs). Predicated on the observations of taking place phenomenon, the detectors ADC converts these analog signals into electronic signs, after which acts as input to your processing apparatus. The processing system comprises chip and also a little storage component that defines the functioning techniques of detector nodes to do the exceptional goal of sensing jobs delegated for them. Transceiver device can be just really actually a relation between also the system and your node. Energy units would be definitely the elements of detector nodes. Energy grubbing components, for example as solar cells may support these.You'll find additional sub par units that can be determined. The understanding of the precise place is called for by the majority of the detection actions and navigation processes from sensor systems. It is an occurrence that is familiar which set locating system is present at a detector node. If some specified delegated tasks must be performed outside, on occasion a mobilizer is required to maneuver tunnels. Even a matchbox-size module ought to have the ability to suit these sub units. The necessary dimension of those sub-units could be bigger compared to the usual centimeter block. That leaves them light in weight that they are able to continue to be suspended in atmosphere. Besides measurement, detector nodes take any delimiting elements that are acute. All these nodes ought to work in densities that are volumetric. They ought to have very lower manufacturing price. Getting autonomous is likewise crucial. They ought to be energy efficient and have to work while swallowing exceptionally very lower ability.

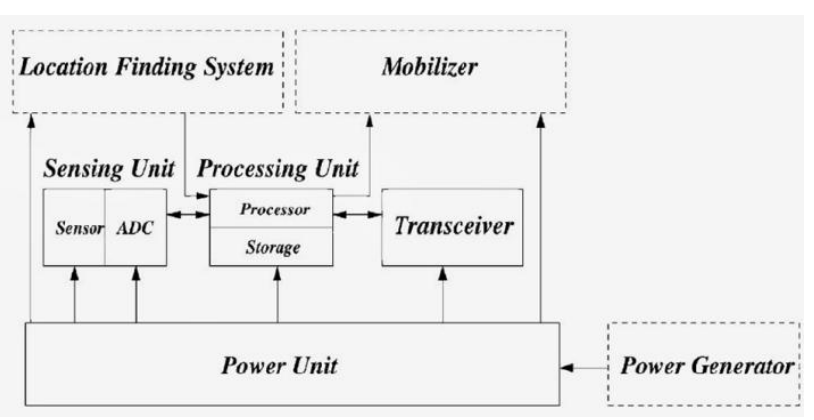

Fig. 1. Components of a sensor node

Wireless Sensor Network

Even a Wireless Sensor Network (WSN) is just really a system using a collection of detector nodes that are connected by radio medium or wireless communicating stations [inch]. The info will be accumulated from your neighboring region by every single detector node that's obviously behaving being a little apparatus, as exemplified sooner. Additionally, it communicates with different detectors nodes or even with all the bottom station (BS) and includes out easy computations too. The detector nodes are by and large deployed in a particular place to track and observe actual time environmental pursuits and also are competent of information transmission and collection into this BS/sink. . Data might be led right back into the spout with multi-purpose jump architecture as shown at Fig.two [inch].The BS/sink can join into this conclusion person or action director node by way of satellite or Internet. The plan of this detector community as clarified by Fig. two depends upon many elements, for example error tolerance, scalability, creation outlays, functioning surroundings and detector system topology, transmission websites and also power-consumption [1].

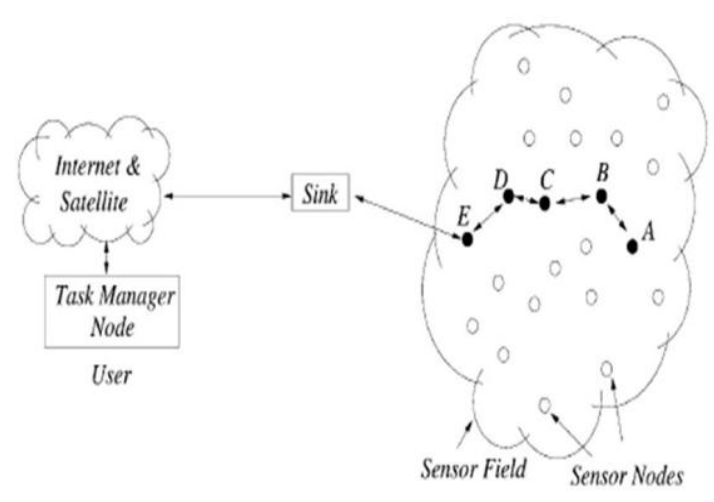

Fig. 2. Communication Architecture of WSN

\section{Protocol Stack}

The routine piles used with the sink and also detector nodes are all awarded at Fig. 3 [1]. The physical coating covers the wants of uncomplicated however powerful modulation, transmission, and even receiving processes. The majority of time, the system atmosphere is noisy and detector nodes will have to become portable and also the moderate access controls (MAC) protocol is to blame for decreasing crash together with nodes that are nearby. The system layer stipulates a mechanism for routing the information offered from the transfer layer.The transfer layer controls the stream of information based to a special detector networks app. Based upon the detection actions, distinct app special applications might be built and assembled to your own applying coating. In addition, the ability direction, mobility management, and undertaking administration airplanes oversee the ability, detector movements and detection task supply one of nodes that are installed. These airplanes defines operations for manipulation of this detection endeavor one of detector nodes and cut back overall energy 
intake. The energy management airplane manages the way the detector node uses its own power.Fig. 3. Protocol StackAs an example, throughout the very minimal degree of energy of detector node, it transmits a note into adjacent nodes it is lower in electricity and can't lead to routing info packets. The rest of the vitality is stored for feeling. The cellular nodes at the detector area are all recognized and farther enrolled from the Cell direction airplane, Which Assists at keeping up a course of the detector nodes, Sensor nodes. Hence detector nodes reach that their mission than many others.

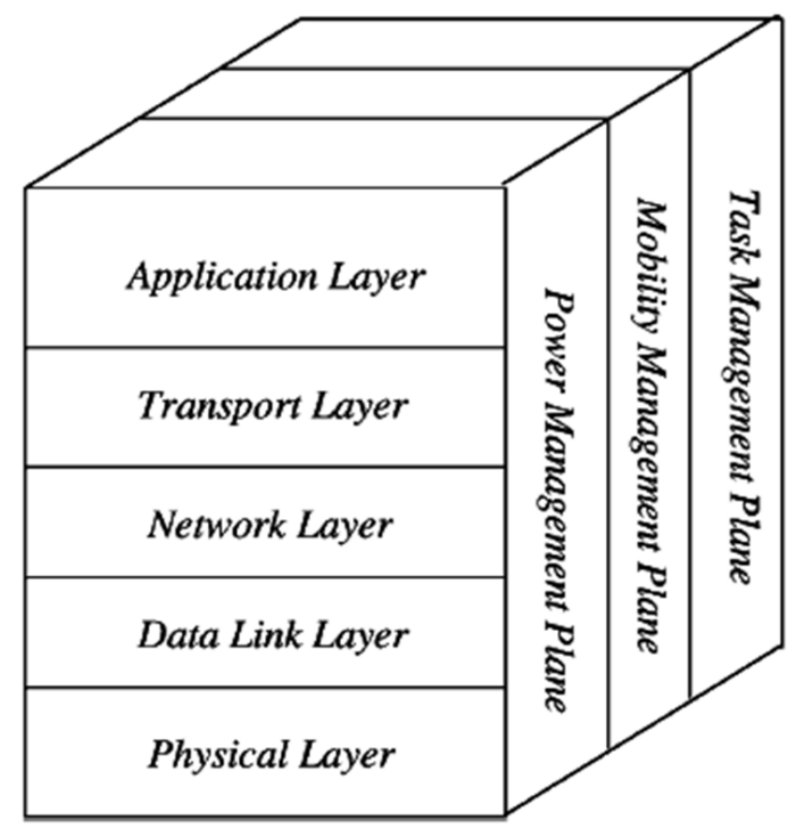

Fig. 3. Protocol Stack

\section{TECHNIQUES FOR ROUTING IN WSNS}

The classification of routing methods may be achieved as: horizontal navigation, hierarchical routing and location-based routing.

- Apartment routing: Every node supposes precisely exactly the job in the network as well as also the detector nodes staff upward to now transport exactly the detection undertaking. The BS maintains searching for advice by the detector when it mailed inquiries to all those 20, found selected regions.

- Hierarchal routes: Cluster established or routing is valuable to scalability in addition to energy-efficiency. Nodes might be used using vitality for sending and processing the info. The synthesis of appointing and clusters activities that are unique to audience heads may result in electricity efficacy and the system scalability.

- Location-based routing. The gap among neighbour nodes are located around the assumption of intensity of signs. In case the detector nodes have been all equipped using a GPS receiver that is tiny, then their location could possibly be reachable forwards.

Outside of these, hierarchical clustering protocols are utilized to increase community and to grow the life of the network by improving power efficacy of every detector node.

\section{BRIEF BACKGROUND}

From WSNs' domain, you can find two communicating protocols: ZigBee and 6LowPAN. 6LowPAN, published in 2007 from IEFT, can be an increasingly open normal conversation protocol about what steps to take to best to utilize IPv6 in addition to low electrical strength, very low data speed, very low priced personal space systems; it performs at the high in both physical and MAC levels, specifying how IPv6 datagrams are sent with 802.15.4 frames by employing compression/decompression of both all IPv6 headers. Additionally, it addresses all the time-varying hyperlink relationship among the nodes containing the WSN. Additionally, it supports the implementation of navigation protocols in the hyperlink coating (net under navigation ) or system coating (path in excess of routing) (J.N.M.Valdez, 2011). BSNs include a detector or set of detectors for gathering information attached. This info processed by manage apparatus, analysed, and will soon probably be transmitted. Even the ZigBee planner as being a commanding apparatus works together with disrupt to decrease energy intake while inside the system -- just a few of many major facets behind health observation -- also collects raw info. Additionally, ZigBee is implemented at a net network of routers to relay info from other patients into the Access Point (AP). The AP is linked to the web allowing cooperation of their medical practioners, health centers, and also different data centers that assemble affected individual documents, and which means that conclusions are available. It might be well worth noting the primary big difference among ZigBee and also 6LowPAN could be that the internet protocol address inter-operability of this latter. Even a ZigBee apparatus demands a open up 802.15.4/ip address gate way to both automatically socialize using an ip address system as a 6LowPAN apparatus communicates with additional IP-enabled apparatus; that you can be preferred tremendously is based upon the applying (C.Buratti, 2009).Detector software will want to join with the internet, $S$ O ZigBee needs to deliver this particular specific feature. Writers in (Sveda and Trchalik, 2007) dedicated to designing applications design amongst ZigBee and also Internet from IEEE 1451. IEEE 1451 is an standard-based media platform which comes with a transducer facts version known as the community Capable Application Processor (NCAP). NCAP can be a integral type that use block lessons, bodily cubes, transducer cubes, perform cubes, and system cubes. ZigBee Gate Way and also ZigBee Bridge have been all proposing to Give connectivity among ZigBee along with also the World Wide Web. Zigbee gate way contrasts controls and speeches in among internet protocol address and also ZigBee. ZigBee Bridge operates above WiFi or Ethernet apparatus and can be utilised to convey with all ip address apparatus. WSN detectors deployed in surroundings and are run with batteries that were modest replacement batteries isn't feasible. Deciding to the battery perhaps maybe not just restricts the detector's life but in addition makes productive style and direction of WSN's a true obstacle. The limit of electricity source has prompted lots of the analysis about WSNs.Community architectures, for example OSI and world 
wide web, are ostensibly operational models structured as levels through which the coating provides products and solutions for the coating over (e.g. the applying form layer gives companies towards the end users). There is A system assessed regarding the product quality like throughput delay, jitter, accessibility, dependability and maybe even safety. But when it regards energy ingestion (EC), you usually faces the issue, as optimization and evaluation of this system as being a detailed version which chooses exactly the EC into consideration scarcely exists. Broadly speaking, investigators concentrate on the conventional network structure and decide to make an effort to reevaluate a particular element of one coating, with all the expectation that the total EC of this system will be paid off with admiration for some different layers or components. This isn't a perfect case, at which you really do not understand a component matches inside of a wireless detector network's total energy arena. Most up to date vitality minimisation versions revolve around receiving and sending info (Wang et al., 2006a), although other parameters have been failed. Back in (Heinzelman et al., 2, 000 ) and (Heinzelman et al., 2002), the energy intake model centered around the price of receiving and sending data and also reevaluate the top limitation of their energy efficacy of single-hop space. In order for your vitality will be saved by the retransmission this method believes an intermediate node in between destination and source. Other procedures rate the power effectiveness of wireless sensor networks using the energy intake model cited previously (Heinzelman et al., 2, 000) and (Heinzelman et al., 2002).They can't suit, As wireless systems have problems and various standards. The cross-layer concept was produced to extend a system design. It's contended by doing this efficiency profits may be gotten in networks, and also the protocols are far appropriate networks when compared with protocols. Broad samples of cross-layer style and layout comprise, state, the design of both either at least two layers collectively, or even passage of parameters involving layers throughout run time, etc..however there's not any requirements to decide on which levels needs to really be united to supply the optimal/optimally effect to your total EC (Mehmet C. Vuran and also Akyildiz).

\section{DISCUSSION\& RESULTS}

WSN detectors deployed in surroundings and are run with batteries that were modest replacement batteries aren't feasible. Deciding to the battery perhaps maybe not just restricts the detector's life but in addition makes productive style and direction of WSN's a true obstacle. The limit of electricity source has prompted lots of the analysis about WSNs.Community architectures, for example OSI and world wide web, are ostensibly operational models structured as levels through which the coating provides products and solutions for the coating over (e.g. the applying form layer gives companies towards the end users). There is A system assessed regarding the product quality like throughput delay, jitter, accessibility, dependability and maybe even safety. But when it regards energy ingestion (EC), you usually faces the issue, as optimization and evaluation of this system as being a detailed version which chooses exactly the EC into consideration scarcely exists. Broadly speaking, investigators concentrate on the conventional network structure and decide to make an effort to reevaluate a particular element of one coating, with all the expectation that the total EC of this system will be paid off with admiration for some different layers or components. This isn't a perfect case, at which you really do not understand a component matches inside of a wireless detector network's total energy arena. Most up to date vitality minimisation versions revolve around receiving and sending info (Wang et al., 2006a), although other parameters have been failed. Back in (Heinzelman et al., 2, 000 ) and (Heinzelman et al., 2002), the energy intake model centered around the price of receiving and sending data and also reevaluate the top limitation of their energy efficacy of single-hop space. In order for your vitality will be saved by the retransmission this method believes an intermediate node in between destination and source. Other procedures rate the power effectiveness of wireless sensor networks using the energy intake model cited previously (Heinzelman et al., 2, 000) and (Heinzelman et al., 2002).They can't suit, As wireless systems have problems and various standards. The cross-layer concept was produced to extend a system design. It's contended by doing this efficiency profits may be gotten in networks, and also the protocols are far appropriate networks when compared with protocols. Broad samples of cross-layer style and layout comprise, state, the design of both either at least two layers collectively, or even passage of parameters involving layers throughout run time, etc..however there's not any requirements to decide on which levels needs to really be united to supply the optimal/optimally effect to your total EC (Mehmet C. Vuran and also Akyildiz).

\section{CONCLUSION}

Successful power usage is really actually a significant factor as the detector node is battery controlled, in deploying wireless sensor systems also it's a life. Research has found that a large part of the capacity is absorbed via info processing systems, information and data detection. Back in WSNsthe tendency will be to incorporate WSNs and web your real-time advice is utilised to create our lifetime as bright as achievable. Due to constrained power supply, one ought to invent a number of innovative power effective routing procedures. This newspaper simplifies hierarchical routing methods predicated on operation parameters also it's discovered the cluster-based systems have a tendency to be much energy effective when compared to other processes.

\section{REFERENCES}

1. L. Buttyan and P. Schaffer, 2007, "PANEL: Position-based Aggregator Node Election in Wireless Sensor Networks," in Proc. of IEEE Internatonal Conference on Mobile Adhoc and Sensor Systems, pp. $1-9$.

2. H. Luo, F. Ye, J. Cheng, S. Lu, and L. Zhang, "TTDD: Two-Tier Data Dissemination in Large-Scale Wireless Sensor Networks," Wireless Networks, vol. 11, no. 1-2, pp. 161-175, 2005. 
3. D. Koutsonikolas, S. Das, Y. C. Hu, and I. Stojmenovic, 2007, "Hierarchical Geographic Multicast Routing for Wireless Sensor Networks," in Proc. of International Conference on Sensor Technologies and Applications, pp. 347-354.

4. A. G. Delavar, S. Shamsi, N. Mirkazemi, and J. Artin, 2012, "SLGC: A New Cluster Routing Algorithm in Wireless Sensor Network for DecreaseEnergy Consumption," International Journal of Computer Science, Engineering and Application, 2(3), pp. 39-51.

5. S. Lindsey, C. Raghavendra, and K. M. Sivalingam, 2002, "Data Gathering Algorithms in Sensor Networks Using Energy Metrics," IEEE Transactions on Parallel and Distributed Systems, 13(9), pp. 924-935.

6. S. Jung, Y. Han, and T. Chung, 2007, "The Concentric Clustering Scheme for Efficient Energy Consumption in the PEGASIS," in Proc. of 9th International conference on Advanced Communication Technology, pp. 260-265.

7. N.Gautam,W.IlLee,andJ.Y.Pyun,2009,"'Track-SectorClus teringforEnergy Efficient Routing in Wireless Sensor Networks," in Proc. of 9th IEEE International Conference on Computer and Information Technology, pp. 116-121.

8. C. Perkins, 2000, Ad Hoc Networks, Addison-Wesley, Reading, MA.

9. J. A. Stankovic, 2004, "Research challenges for wireless sensor network" ACM SIGBED Review- Special issue on embedded sensor networks and wireless computing, 1(2), pp. 9-12.

10. Akkaya and M. Younis, 2005, "A Survey on Routing Protocols for Wireless Sensor Networks," Ad Hoc Networks, 3(1), pp. 325 - 349.

11. B. Krishnamachari, 2005, Networking Wireless Sensors, Cambridge University Press.

12. G.Hoblos,M.Staroswiecki,A.Aitouche,2000, “Optimaldes ignoffaulttolerant sensor networks," IEEE International Conference on Control Applications, Anchorage, AK, pp. 67-472.

13. D. Nadig, S.S. Iyengar, 1993, "A new architecture for distributed sensor

14. C. Shen, C. Srisathapornphat, C. Jaikaeo, 2001, "Sensor information networking architecture and applications," IEEE Personal Communications, pp. 52-59.

15. S. Cho, A. Chandrakasan, 2000, "Energy-efficient protocols for low duty cycle wireless microsensor," Proceedings of the 33rd Annual Hawaii International Conference on System Sciences, Maui,HI, vol. 2 , pp. 10.

16. N. Bulusu, D. Estrin, L. Girod, J. Heidemann, 2001, "Scalable coordination for wireless sensor networks: self-configuring localization systems," International Symposium on Communication Theory and Applications (ISCTA 2001), Ambleside, UK.

17. J.M. Rabaey, M.J. Ammer, J.L. da Silva Jr., D. Patel, S. Roundy, 2000, "PicoRadio supports ad hoc ultra-low power wireless networking, "IEEE Computer Magazine, pp. $42-48$

18. J. Rabaey, J. Ammer, J.L. da Silva Jr., D. Patel, 2000, "Pico-Radio: ad-hoc wireless networking of ubiquitous low energy sensor/monitor nodes," Proceedings of the IEEE Computer Society Annual Workshop on VLSI (WVLSI'00), Orlanda, Florida, pp. 9-12.

19. FengyuanRen, J. Z., Tao He, Chuang Lin, Sajal K. Das, December, 2011 EBRP: Energy- Balanced Routing Protocol for Data Gathering in Wireless Sensor Networks. IEEE Transactions on Parallel and Distributed Systems, 22, 2108-2125

20. Foster Provost, T. F. 2013. Data Science for Business: What you need to know about data mining and data-analytic thinking, O'Reilly Media.

21. G. Virone, A. W., L. Selavo, Q. Cao, L. Fang, T. Doan, Z He, And J. A. Stankovic 2006. An advanced wireless sensor network for health monitoring. Distributed
Diagnosis and Home Healthcare.

22. Garcia-Luna-Aceves, J. J., Mosko, M. \& Perkins, C. E. 2006. A new approach to on-demand loop-free routing in networks using sequence numbers. Comput. Netw., 50, $1599-1615$

23. Ghaffari, A. 2014. An Energy Efficient Routing Protocol for Wireless Sensor Networks using A-star Algorithm. Journal of Applied Research and Technology, 12, 815-822.

24. Goldsmith, A. J. \& Wicker, S. B. 2002. Design Challenges for Energy-Constrained Ad Hoc Wireless Networks. IEEE Wireless Communications Magazine, 8-27.

25. H.Edgar\& Callaway, J. 2004. Wireless Sensor Networks.

26. Halkes, G. P., Dam, T. \&Langendoen, K. G. 2005 Comparing energy-saving MAC protocols for wireless sensor networks. Mobile Networks and Applications Journal of Kluwer Academic, 10, 783-791.

27. Hall, M. A. \& Smith, L. A. 1999. Feature Selection for Machine Learning: Comparing a Correlation- Based Filter Approach to the Wrapper. Proceedings of the Twelfth International Florida Artificial Intelligence Research Society Conference. AAAI Press.

28. Hande, A. \&Cem, E. 2010. Wireless sensor networks for healthcare: A survey. Comput. Netw.\%@1389-1286, 54, 2688-2710

29. Hastie T, T. R., Friedman J 2009. The Elements Of Statistical Learning: Data Mining, Inference, and Prediction, Springer.

30. Heinzelman, W. 2000. Application Specific Protocol Architectures for Wireless Networks. Ph.D Thesis, Massachusetts Institute of Technology.

31. Heinzelman, W., Kulik, J. \&Balakrishnan, H. Adaptive Protocols for Information Dissemination in Wireless Sensor Networks. 5th ACM/IEEE Mobicom Conference August 1999a Seattle, WA. 174-85.

32. Heinzelman, W. R., Chandrakasan, A. \&Balakrishnan, H 2000. Energy-efficient communication protocol for wireless microsensor networks. 33rd Annual Hawaii International Conference on System Sciences. 